\title{
Wie der mensch seinen leib in gesundheyt behalten sol Die mittelalterlichen Gesundheitslehren am Übergang vom Lateinischen ins Deutsche
}

\author{
Melitta Weiss ADAMSON
}

\begin{abstract}
Wie der mensch seinen leib in gesundheyt behalten sol. Medieval guides to healthy living at the transition from Latin to German

With the preservation of health an age-old concern for humanity, guides to healthy living based on humoral theory were among the earliest texts of medieval school medicine to be translated from Latin into the vernacular. Subject of this study is the development of a German technical language for dietetics from the late thirteenth to the late fifteenth century as evidenced in Hiltgart von Hürnheim's translation of the 'Secretum secretorum', the anonymous translation of the regimen in the 'Breslauer Arzneibuch', and the four independent translations of Konrad von Eichstätt's 'Regimen sanitatis'. Special emphasis is put on a number of termini technici from humoral theory and the way the various translators tackled these terms.
\end{abstract}

Keywords: late Middle Ages, regimen sanitatis, vernacularization, German technical language, humoral theory

DOI: doi.org/10.15452/StudiaGermanistica.2020.26.0001

\section{Einleitung}

Die Erhaltung der Gesundheit ist seit Jahrtausenden ein Anliegen der Menschheit und avancierte im Mittelalter zu einem bedeutenden Bereich der Medizin. Mittelalterliche Gesundheitslehren, bekannt unter der Bezeichnung regimina sanitatis, waren der Medizin der Antike, speziell den Werken von Hippokrates und Galen, verhaftet. Sie basierten auf der Humorallehre und benutzten die vom Menschen beeinflussbaren Faktoren, sex res non naturales genannt, nämlich Luft (aer), Bewegung und Ruhe (motus et quies), Essen und Trinken (cibus et potus), Schlafen und Wachen (somnus et vigilia), Füllung und Leerung (repletio et evacuatio) und Gemütsbewegungen (accidentia animi) als Ordnungsprinzip. Von den Arabern im Frühmittelalter zu einem wichtigen Genre der medizinischen Literatur erhoben, erreichten die Gesundheitslehren im Hochmittelalter Europa, in erster Linie über Italien und das Corpus Constantinum, die lateinischen Übersetzungen aus dem Arabischen von Constantinus Africanus, die in Salerno gelehrt wurden, und über Spanien und das Corpus Toletanum, die lateinischen Übersetzungen aus dem Arabischen, die in Toledo angefertigt und in Montpellier und Paris gelehrt wurden (Schmitt 1970). In der Theorie der Medizin der Zeit bildeten die res non 
naturales das Bindeglied zwischen den res naturales und den res contra naturam (ibid.:79). Die Übersetzung diätetischer Literatur vom Lateinischen in die europäischen Volkssprachen begann im Fall von Deutschland erstaunlich früh und zeugt von einem intensiven Interesse von Laien an Ratgebern für ein gesundes Leben.

Bereits im späten 13. Jahrhundert wurde von der Nonne Hiltgart von Hürnheim eine deutsche Fassung des ,Secretum secretorum' angefertigt, das ein Regimen enthält (Möller 1963). Im frühen 14. Jahrhundert folgte die Übersetzung des Regimens im ,Breslauer Arzneibuch', das hauptsächlich auf dem Regimen im ,Liber pantegni“ von Haly Abbas basierte (Külz und Külz-Trosse 1908). Zur gleichen Zeit kompilierte der deutsche Arzt Konrad von Eichstätt ein lateinisches ,Regimen sanitatis', das nicht nur zu einer, sondern vier separaten Übersetzungen ins Deutsche führte, die zwischen der zweiten Hälfte des 14. Jahrhunderts und dem letzten Viertel des 15. Jahrhunderts entstanden und heute unter den Titeln ,Regimen vitae“ (Hagenmeyer 1995:193-222), ,Regel der Gesundheit" (Strauss 1963), ,Ordnung der Gesundheit“ (Hagenmeyer 1972) und ,Büchlein der Gesundheit" (Hagenmeyer 1995:223-254) bekannt sind. Auch Heinrich Laufenbergs ,Versehung des leibs' von 1429, das in Versen abgefasst ist, dürfte Konrads ,Regimen' als Quelle benutzt haben (Menge 1976, Schnell 1998:20). Das berühmte ,Regimen sanitatis Salernitanum‘, ebenfalls in Versen, zirkulierte im Spätmittelalter in hoch- und niederdeutscher Übersetzung, sowie in vielen anderen europäischen Volkssprachen (Schmitt 1970:12). Im Folgenden soll die Entstehung einer deutschen Fachsprache für Diätetik in den zwei Jahrhunderten des ausgehenden Mittelalters untersucht werden, wobei die mehrfachen Übersetzungen von Konrads ,Regimen sanitatis` und die Art und Weise, wie die verschiedenen Übersetzer eine Reihe von termini technici aus der Humorallehre behandelten, im Mittelpunkt stehen. Dabei ist festzuhalten, dass in der Folge repräsentative Übersetzungsbeispiele gegeben werden, die keinen Anspruch auf Vollständigkeit erheben. Die Erfassung des gesamten diätetischen Fachvokabulars des Textkorpus inklusive Schreibvarianten hätte den Rahmen dieser Studie gesprengt.

\section{Das ,Secretum secretorum'}

Das ,Secretum secretorum', das vorgibt, eine Sammlung von Briefen zu sein, die Aristoteles seinem berühmten Schüler Alexander dem Großen geschrieben haben soll, ist eine vermutlich aus dem 10. Jahrhundert stammende arabische Enzyklopädie, die im Orient in zwei Versionen Verbreitung fand, einer kürzeren westlichen und einer längeren östlichen Version (Adamson 1995: 50f). Beide erreichten Europa und wurden ins Lateinische übersetzt. Es war die längere Version, die Hiltgart von Hürnheim im späten 13. Jahrhundert als Basis für ihre deutsche Prosaübersetzung diente. Neben Staatsführung, Ethik, Astrologie, Alchemie und Physiognomie befasst sich der größte Teil der Briefe, an die vierzig Prozent, mit der Erhaltung der Gesundheit. Obwohl der diätetische Inhalt nicht nach den sex res non naturales angeordnet ist, werden alle sechs auf verschiedene Weise in den Regimen-Abschnitten behandelt, die in Möllers Edition die Kapitel 27 bis 53 umfassen (Möller 1963:58-99). Was die Terminologie der res non naturales betrifft, so besteht bereits im lateinischen Text eine gewisse Verwirrung, die in der deutschen Übersetzung ebenfalls zu beobachten ist: Kapitel 27 spricht vom Wissen der rerum naturalium genera, was sich nicht unbedingt auf die res non naturales beziehen muss, und von Hiltgart wortgetreu als der natürlichen dinge übersetzt wird (ibid.:58f.). Was später in dem Kapitel folgt, ist eine unvollständige Liste der res non naturales, die mit lose damit verbundenem Material, wie etwa dem Aderlass, erweitert ist: in pleno et in vacuo, in sompno et in vigiliis, in motu et in quiete, in dissolutione et retentione ventris, in subtractione vel detentione sanguinis (ibid.:60). Auch hier hält sich die Übersetzerin wieder eng an ihre lateinische Quelle: an der völle unnd an eitelm, an schlaffe und an wachen, an pewegde und an rue, an waiche und an hertte des leibes, an vil lassen unnd an wenigk lassenn (ibid.:61). Kapitel 46, De oculis, endet mit der Nennung der sex res non naturales und bringt nahezu die gesamte Liste: Observetur cum hoc regiminis bonitas in sex res non (?) naturalibus: aer, exercicio et ocio, cibo et potu, somno et vigilia, coitu et accidentibus anime (ibid.:86). Hiltgart übersetzt die Passage wie folgt: Die güete diser 
richtegung ist an sechs natürlichen dingen: an dem lufft, an der übung unnd an der müessigkait, an essenn unnd an trincken, an schlaffen unnd an wachen, unnd an unkeüsch, und an den zu treffenden dingen der sele (ibid.:87).

Was die lateinische Terminologie betrifft, die sich auf die Humorallehre bezieht, so übernimmt die Übersetzerin manchmal die Wörter aus ihrer Quelle, wie das etwa bei complexio oder temperantia der Fall ist, die als complexion oder temperung wiedergegeben werden (ibid.:58 f.). Für andere bringt sie eine Übersetzung, wie zum Beispiel für superfluitas das deutsche Wort überflüssigkhait (ibid.:60, 63). Die vier Temperamente werden im diätetischen Teil des ,Secretum' nicht behandelt, nur einmal spricht der Text von in colerico signo, von Hiltgart als in dem colerischen zaichenn übersetzt (ibid.:108, 111). Die Termini humores und humiditas werden wiederholt im Text gebraucht, aber anstatt zu differenzieren, verwendet Hiltgart feüchte für beide (ibid.:58 f., 62 f.). Die wenigen Autoritäten, die im Regimen des ,Secretum' genannt werden, sind auch in der Übersetzung enthalten. Kapitel 57 bringt neun Rezepte für zusammengesetzte Drogen, die allerdings unübersetzt bleiben. Das deutet darauf hin, dass die Zielgruppe für die Drogen eine andere war als für den Diätetikteil mit seinen allgemein gehaltenen Ratschlägen, man denke hier etwa an Apotheker (ibid.:101-106). Wie Reinhold Möller, der Herausgeber von Hiltgarts deutscher Fassung des ,Secretum', in seiner linguistischen Analyse bemerkt, besaß die Übersetzerin gute Lateinkenntnisse und dürfte mit ihrer deutschen Version der Quelle getreu gefolgt sein (ibid.:CII).

\section{3. ,Das Breslauer Arzneibuch ${ }^{6}$}

Kurz nachdem Hiltgart ihr Werk beendet hatte, wurde eine weitere deutsche Übersetzung eines diätetischen Texts in einen Pergamentkodex in Breslau eingetragen, der heute als das ,Breslauer Arzneibuch' bekannt ist (Külz und Külz-Trosse 1908, Adamson 1995:103-109). Das Werk behandelt sowohl Vorsorge als auch Heilung und ist eine Kompilation verschiedener Traktate, die auf Salerno als Entstehungsort weisen. Den Auftakt bilden Abschnitte über die vier Elemente und die vier Säfte, die unbekannter Herkunft sind (Külz und Külz-Trosse 1908:1-7). Darauf folgen Auszüge aus dem regimen sanitatis im ,Liber pantegni“ von Haly Abbas, wobei speziell Essen und Trinken, Schlafen und Wachen, Koitus und Baden behandelt werden. Die beiden letztgenannten sind bei Haly Abbas Teil des Abschnitts inanitio et repletio (ibid.:7-26, Adamson 1995:44). Die Fachsprache der Humorallehre wird vom deutschen Übersetzer gleich zu Beginn des ,Arzneibuchs', in der Abhandlung über die vier Elemente und vier Säfte, eingeführt (ibid:.1-7). Für die vier Elemente der Welt verwendet er den lateinischen Terminus im Plural, elementa, aber für die Elemente selbst benutzt er die deutschen Ausdrücke vuer, luft, wazzer und erde (ibid.:1). Wie in Hiltgarts Übersetzung erscheinen die vier Säfte als vier vuchtin (ibid.). Von denen wird nur eine ins Deutsche übersetzt, nämlich blut, während die anderen drei ihre lateinischen Formen colera, flecma und melancolia beibehalten (ibid.). Der Text informiert den Leser, dass die vier Säfte im menschlichen Körper durch Essen und Trinken erzeugt werden und dass jeder in der richtigen Balance naturlich ist, und wenn die Balance verlorengeht, Daz vnnaturliche ist daz die nature nich rechte getempert hat (ibid.:2). Wie bei Hiltgart wird auch hier der Stamm des lateinischen Verbs temperare beibehalten und später die Nominalform temperunge verwendet (ibid.:6). Eine Diskussion über die vier Säfte blut, fleuma, colera und melancolia in ihrer ,natürlichen“ und „unnatürlichen“ Form, ihr Fluktuieren in den verschiedenen Monaten des Jahres und Stunden des Tages wird gefolgt von einer Zuordnung der Säfte zu den Sinnesorganen Nase, Ohren, Augen und Gaumen und der Auswirkung der Säfte auf den Charakter der Menschen (ibid.:2-5). Erst gegen Ende des Traktates werden die vier Primärqualitäten warm, kalt, feucht und trocken als uir qualitates bezeichnet und der Terminus complexio wird eingeführt und erklärt: Complexio heize wir di da wirt uon der uir qualitatum mischunge (ibid.:6). Da das Regimen von Haly Abbas nicht nur über die Primärqualitäten informiert, sondern auch über ihre Intensität vom ersten bis zum vierten Grad, und fallweise sogar eine weitere Unterteilung eines Grades in Anfang, Mitte und Ende, schließt der Traktat mit einer Einführung in diese Klassifizierung (ibid.:6-7). Dass der Übersetzer dabei den lateinischen Terminus gradus beibehält, sollte nicht 
überraschen, wobei er dann auf Deutsch weiter differenziert zwischen erstin, andirn, dritten und uirden gradu, und innerhalb eines Grades zwischen anuenge, mitter und daz ende. Der Terminus superfluitas, der im ,Breslauer Arzneibuch“ im Zusammenhang mit Koitus und Baden erscheint, wird, wie bei Hiltgart, mit uberuluzekeit übersetzt (ibid.:23, 25).

\section{Das ,Regimen Sanitatis' Konrads von Eichstätt}

Im frühen 14. Jahrhundert, vermutlich zwischen 1310 und 1320, kompilierte der deutsche Arzt Konrad von Eichstätt ein Regimen mit Material, das er in erster Linie den Werken von Avicenna, Rasis und Averroës entnahm und das zur Matrix für die deutsche Regimenliteratur werden sollte (Hagenmeyer 1995:64-118, Adamson 1995:142-149). Im Umlauf waren zwei Versionen, eine längere, das sogenannte ,Urregimen', und eine kürzere, der ,Sanitatis Conservator', die im deutschen Sprachraum eine enorme Reichweite hatten, besonders nachdem die deutschen Übersetzungen und der Buchdruck sie breiten Kreisen der Bevölkerung zugänglich und erschwinglich machten. Nach Christa Hagenmeyers Berechnungen war allein die in der ,Ordnung der Gesundheit ${ }^{*}$ enthaltene deutsche Übersetzung zwischen 1400 und 1600 im hochdeutschen Sprachgebiet in mindestens zehn tausend Kopien vorhanden (Hagenmeyer 1995:177).

Konrad, der vermutlich ein Absolvent der medizinischen Fakultät in Montpellier war, was aus seinen Quellen ersichtlich wird, die er in seinem ,Urregimen' akribisch zitiert, inklusive Autor, Titel des Buches oder Traktats und oft auch Kapitel, ist mit der Humorallehre und dem Konzept der res non naturales bestens vertraut. Er zitiert sie gleich in der Einführung als sex res non naturales, scilicet motus et quies, cibus et potus, somnus et vigilia, aer, et inanitio et repletio, accidentia animi (ibid.:65). In seinem Regimen selbst befasst er sich allerdings nur mit den ersten fünf. Wie viele europäische Regimenautoren übergeht er die accidentia animi, ein Thema, das im Mittelalter in der arabischen Welt offensichtlich einen höheren Stellenwert besaß als im christlichen Europa. Unter De inanitione et repletione finden sich bei Konrad neben Baden, Koitus und Erbrechen auch Abschnitte über Aderlass und Klistiere (ibid.:78-93). Dem allgemeinen Regimen angeschlossen ist sodann eine Lebensmitteldiätetik (ibid.:93-118).

\subsection{Das ,Regimen vitae}

Die früheste deutsche Übersetzung von Konrads Regimen aus der zweiten Hälfte des 14. Jahrhunderts ist das ,Regimen vitae', das sowohl das allgemeine Regimen als auch die Lebensmitteldiätetik enthält und wie alle anderen Übersetzungen auf dem längeren ,Urregimen` basiert, ohne allerdings den Namen des Autors/Kompilators zu nennen (Hagenmeyer 1995:193-222). Nicht enthalten sind die Abschnitte über Aderlass und Klistiere, sowie in der Nahrungsmitteldiätetik die Gewürze. Konrads Kapitel über den Koitus und eine Handvoll lateinischer Verse aus Avicennas ,Cantica canticorum' und aus dem ,Regimen sanitatis Salernitanum' sind allerdings erhalten geblieben. Einige neue, weniger kostspielige Zutaten wurden am Ende der Lebensmitteldiätetik anstelle der Gewürze hinzugefügt, was auf ein etwas bescheideneres Publikum als Zielgruppe deuten könnte (Adamson 1995:177). Das ,Regimen vitae‘ wurde in Verbindung mit dem ,Arzneibuch“ Ortolfs von Baierland und dem ,Kräuterbuch“ Konrads von Megenberg tradiert und als Teil dieses Textkorpus in sieben Inkunabelausgaben gedruckt (Hagenmeyer 1995:180-183).

Wie Hiltgart und der Übersetzer des ,Breslauer Arzneibuchs“ hat auch der Übersetzer des ,Regimen vitae" Probleme mit den sex res non naturales. Er nennt sie sechs sach und identifiziert sie als die måßlich wegen des leibs, růe, speis und tranck, schlaf und wachen, mit anderen Worten, gemäßigte Bewegung des Körpers, Ruhe, Essen und Trinken, Schlafen und Wachen (ibid.:193). Der Übersetzer zählte offensichtlich jeden Teil der Gegensatzpaare einzeln, wodurch er de facto nur drei der sex res non naturales auflistet. Bei der Terminologie aus der Humorallehre benutzt er eine ähnliche Strategie wie die früheren zwei Übersetzer, nämlich die lateinischen Termini soweit wie möglich beizubehalten. Und so bleibt er beim Terminus complexio, den er folgendermaßen erklärt: 
complexen, das ist nach siner art (ibid.:196). Die vier Temperamente werden ebenfalls unverändert und mit dem lateinischen Dativ als dem melancolico, dem colerico, dem sanguineo und dem flegmatico wiedergegeben (ibid.). Für den lateinischen Ausdruck temperamentum wird einmal temperierung benutzt (ibid.:211), für das Verb temperare üblicherweise temperieren, sowie das Partizip Perfekt getemperirt (ibid.:194, 196, 200). Das Adverb temperate wird auch bisweilen als måßlich übersetzt (ibid.:201). Das Substantiv superfluitas erscheint als überflüssigkeyt (ibid.:202) oder allgemein als bose feuchte (ibid.:209), ein Terminus, der im ,Regimen vitae eine weitaus größere Zahl von Ausdrücken umfasst als die früheren Übersetzungen. Er wird für humores (ibd.:200), humiditas (ibid:201), flegmaticas (ibid.:212) und sogar für multa nocumenta (ibid.:200) gebraucht. Ein weiterer Sammelbegriff ist bo $\stackrel{e}{\text { ses }}$ plüt, der melancolicus sanguis, flegmaticus sanguis (ibid.:210f.) oder einfach sanguis malus (ibid.:213) bedeuten kann. Für colera rubea und colera nigra werden nur die Adjektive als rot und schwartz ins Deutsche übersetzt (ibid.:199f.). Die Namen der medizinischen Autoritäten in Konrads Text werden in der deutschen Version beibehalten, Buchtitel und Kapitelverweise wurden jedoch entfernt.

\subsection{Die ,Regel der Gesundheit'}

Die ,Regel der Gesundheit‘ (Strauss 1963) ist eine Kompilation aus drei lateinischen regimina in deutscher Übersetzung, dem ,Regimen sanitatis` von Gregorius (Ferckel 1918:5-20), dem ,Regimen sanitatis ad inclitum dominum regem Aragonum“ von Arnald de Villanova (García-Ballester et al. 1996:423-470) und Konrads ,Regimen sanitatis' (Hagenmeyer 1995:64-118). Anstatt das Material aus den verschiedenen Quellen zu integrieren, führt der Kompilator die Exzerpte nacheinander an, was viele Überlappungen zur Folge hat. Nachdem Konrads Passagen zuletzt erscheinen, ist es auch denkbar, dass sie später hinzugefügt wurden, was die Datierung dieser Übersetzung von seinem Regimen erschwert. Die älteste Handschrift der , Regel ${ }^{`}$, die Konrads Auszüge enthält, ist auf 1421 datiert, könnte aber bereits einige Jahrzehnte früher übersetzt worden sein (Hagenmeyer 1995:183 f., Adamson 1995:180-190). Im Gegensatz zum ,Regimen vitae“ wurde die ,Regel der Gesundheit", die in zwölf Handschriften erhalten ist, nie gedruckt. Sie wurde von einem Adeligen in Auftrag gegeben und in der ersten Hälfte des 15. Jahrhunderts von Meister Eberhard, einem Koch im Dienste des Adels, dazu benutzt, sein Kochbuch mit diätetischen Informationen anzureichern (Feyl 1963, Hirth 1966). Da die ,Regel' mit Material aus drei Regimentexten kompiliert wurde, nennt sie nicht nur die sex res non naturales, sondern behandelt sie auch, angefangen mit den accidentia animi, gerade dem Kapitel, das bei Konrad fehlt. Bei den weiteren fünf res non naturales erweist sich dann allerdings Konrads ,Regimen' als Hauptquelle, wobei für Essen und Trinken sowohl die allgemeinen Richtlinien als auch die Lebensmitteldiätetik übernommen werden. Wie im, Regimen vitae" wurden die detaillierten Zitate der lateinischen Fassung radikal reduziert, in diesem Fall primär auf die Nennung von Autoren, wobei Almansor auch als Autor und nicht als Empfänger von Rasis',Regimen ' betrachtet wird.

In seiner Einführung der sex res non naturales bezeichnet der Übersetzer sie als sechs sach, die nützlich sein können oder schädlich, wenn man sich nicht entsprechend daran hält, und er nennt die fünf, die von Konrad tatsächlich behandelt werden, nämlich ubunge oder erbeit vnd rohe, spy $\beta$ vnd trang, slaffen vnd wachen, zu vol oder tzu wan vnd der luffte etc. (Strauss 1963:91). Der lateinische Terminus complexio wird als complexien beibehalten (ibid.:98, 109), jedoch in Verbindung mit calida oder frigida übersetzt mit hytzigen oder kalten luten (ibid.:139). Das Wort fuchtikeit wird auch in diesem Regimen für eine Reihe lateinischer Termini gebraucht, für humores (ibid.:121, 123, 136 f.), humiditas (ibid.:119, 141), humectare (ibid.:121) und superfluitas (138, 140). Anfänglich erscheinen flegma, melancolia und colera noch in dieser Form und nur sanguis wird mit blut übersetzt (ibid.:109), aber in der Folge kommt es zu einem allmählichen Übergang von colera zu vbergelle, im Speziellen bei colera rubea (ibid.:123, 127 f., 134). Weiters werden die melancolici im Deutschen zu durren kalten leuten (ibid.:142), die colerici zu hitzigen leuten (ibid.) und aus humor melancolicus wird boß blut (ibid.:135). Die Tendenz, lateinische Termini zu erklären und schließlich mit deutschen zu ersetzen ist in diesem Regimen stärker als in den vorhergehenden und wird 
vielleicht am besten dadurch illustriert, dass Ausdrücke wie temperare, temperatus oder temperantia verschwunden sind. An ihre Stelle treten Wörter wie bescheidenlich (ibid.:129, 134, 138) oder gut (ibid.:123 f., 127), und für das Gegenteil, distemperate, der Terminus vnnatürlich (ibid.:130).

\subsection{Die ,Ordnung der Gesundheit ${ }^{6}$}

Die dritte Übersetzung von Konrads Regimen ist Teil der ,Ordnung der Gesundheit', die um 1400 von Rudolf VI. von Hohenberg in Auftrag gegeben wurde und in der Folgezeit viel weitere Kreise als den Adel erreichte, wie zum Beispiel Kleriker, Ärzte und Barbiere, sowie gebildete Bürger (Hagenmeyer 1972, Adamson 1995:161-169). In einem Fall wurde die ,Ordnung ' sogar ins Lateinische rückübersetzt (Hagenmeyer 1995:179). Ähnlich dem ,Breslauer Arzneibuch“ behandelt das erste Buch so allgemeine Themen wie die Jahreszeiten, eine Monatsregel, die Elemente und Temperamente. Darauf folgt der erste Teil von Konrads Regimen in deutscher Übersetzung, der sich mit fünf der sechs res non naturales befasst. Für Essen und Trinken werden nur die allgemeinen Ernährungsratschläge gebracht und nicht Konrads Lebensmitteldiätetik. Konrads Regimen erscheint gestrafft und ist unterteilt in 153 Einzelkapitel. Während einerseits seine Abhandlung über den Koitus fehlt, erscheint andererseits der Aderlass mit neuem Material angereichert (ibid.:178).

Für die vier Elemente und ihre Primärqualitäten verwendet der Übersetzer die deutschen Ausdrücke erd, wasser, luft und fuir, sowie respektive kalt, feucht, trucken und warm (ibid.:289), übernimmt aber bei den Temperamenten die lateinischen Termini melancolicus, flegmaticus, sangwineus und colericus (ibid.:290, 295) und gibt complexio als conplexion wieder (ibid.:291). Bei den sex res non naturales ist die deutsche Fassung sehr vage: mit essen und trincken, mit schlaffen, baden, oder lassen und mit andern dingen, die hernach geschriben stand (ibid.:292). Für humores und humiditas wird wieder feichtikayt verwendet (ibid.:294), während humectatio als feucht machen übersetzt wird (ibid.:306). Superfluitas erscheint auch hier als überflussikait (ibid.:298, 309), und bei colera rubea (ibid.:303, 307, 311) und colera nigra (ibid.:304) wird nur jeweils das Adjektiv ins Deutsche übersetzt. Für temperatus bringt der Übersetzer der Ordnung deutsche Ausdrücke wie etwa messig (ibid.:295), messlichen (ibid.:305), messiclichen (ibid.:304), oder zerechter maß (ibid.:296). Das Verb temperare erscheint nur einmal als temperiern im Regimen sanitatis (ibid.:297), aber häufiger im nachfolgenden Pesttraktat (ibid.:233-242). Allgemein ist zu beobachten, dass der Übersetzer gerne seinem Publikum Erklärungen für lateinische Ausdrücke gibt, die ihm möglicherweise unbekannt waren, wie zum Beispiel ethica, das ist das abnemen (ibid.:312), evacuacio, das ist die rainigung (ibid.:327 f.), oder vomitus, das ist das brechen (ibid.:328). Für letzteren Terminus erfindet er später das Verb vomiten und schreibt: So der mensch vil vomitet, das ist sich prech (ibid.:329). Die Tatsache, dass im Laufe des Regimens in der Ordnung immer mehr lateinische Termini beibehalten werden, könnte auch auf einen Wechsel mitten im Text zu einem anderen Übersetzer deuten.

\subsection{Das ,Büchlein der Gesundheit}

Eine ähnliche Struktur wie die ,Ordnung der Gesundheit' weist die medizinische Kompilation auf, die die vierte unabhängige deutsche Übersetzung von Konrads Regimen beinhaltet, das ,Büchlein der Gesundheit' (Hagenmeyer 1995:223-254). Es wird auf das letzte Viertel des 15. Jahrhunderts datiert und ist nur in einer Handschrift erhalten. Seinen eigenen Worten zufolge war der Kompilator auch der Übersetzer dieses Auftragswerkes, das mit einer Abhandlung über die vier Jahreszeiten beginnt, gefolgt von einer Monatsregel, den Elementen und Temperamenten, bevor es sich Konrad als Quelle zuwendet. Nicht enthalten in dem Abschnitt repletio et evacuatio sind die Kapitel über Purgieren, Koitus und Klistiere, während andererseits eine Hämatoskopie neu hinzugekommen ist. Was cibus et potus betrifft, so sind sowohl die allgemeinen Ratschläge als auch die Lebensmitteldiätetik enthalten, aber wie im Fall des ,Regimen vitae' wurden die Gewürze von letzterer entfernt. Erhalten geblieben sind Konrads detaillierte Quellenangaben, was diesen Text einzigartig unter den deutschen Konradübersetzungen macht (ibid.:186-191). 
Vieles bei der Behandlung der termini technici im ,Büchlein“ ist den bisher genannten Übersetzungen ähnlich. Complexio erscheint als complexion (ibid.:227, 230), die vier Temperamente erscheinen als melancolicus, colericus, sangwineus und flegmaticus (ibid.:227), und die sex res non naturales sorgen leider auch bei diesem Übersetzer für Verwirrung. Er spricht von dingen und erklärt wie folgt: Der sein segs, nemlich arbait, rue, essen, trincken, schlaffen, wachen. Und etlich settzen hintzu den luft wan wie wenig oder wie vil (ibid.:224). Feuchtikait wird für humiditas (ibid.:224) und humor (ibid.:226, 231, 239) verwendet, und feucht zu machen für humectatio (ibid.:232). Superfluitas erscheint als uberflussikait (ibid.:224, 228). Das Adjektiv temperatus wird als maslich übersetzt (ibid.:233), während der lateinische Stamm in dem deutschen Verb temperirn (ibid.:239,241) und der Partizip Perfekt Form getemperiert erhalten ist (ibid.:242, 246-248, 251). Für colera rubea gibt er die Standardübersetzung rot colera und erklärt danach daz ist hittzsucht (ibid.:230, 241). Worterklärungen finden sich auch in dieser Übersetzung, so etwa ethica, das ist in rechtem teutsch das abnemen (ibid.:224). Die Tendenz, Termini zu erklären, ist im ,Büchlein“ allerdings etwas weniger ausgeprägt als in der ,Ordnung'.

\title{
5. Fazit
}

Zusammenfassend ist festzuhalten, dass die von den Übersetzern der verschiedenen lateinischen regimina angewandten Strategien sehr ähnlich sind, was die Terminologie der Humorallehre betrifft. Sie behalten oft die lateinischen Termini bei, die sie in ihren Quellen vorfinden, mit oder ohne Erklärung derselben, sie verwenden des weiteren gern vage Sammelbegriffe wie bo $\stackrel{e}{s}$ plüt oder bos fuchtikeit, womit sie sich detaillierte Erklärungen für Begriffe aus der Humorallehre ersparen, sie geben Teilübersetzungen wie etwa rot colera, und gehen nur langsam zu deutschen Termini über, wie vbgergelle für colera. Alle Übersetzer sind verwirrt über die sex res non naturales und worum es sich dabei genau handelt. In Unkenntnis der Theorie der Medizin dürfte es ihnen widersinnig erschienen sein, so natürliche Aktivitäten wie Essen und Schlafen als ,nicht natürlich' zu bezeichnen, ebenso wie Gegensatzpaare als jeweils ein res non naturalis zu betrachten. Erst Heinrich Laufenberg, einem Kleriker, ist es vorbehalten, in seiner, Versehung des leibs‘ von 1429 als erster alle sechs stúken mit ihrer korrekten Zuordnung als nit natúrlich aufzulisten:

\author{
Nit natúrlich heissent sy \\ Das erste ist Vibunge vnd hie by \\ Ist das ander trinken vnd essen \\ Schloffen wachen nit vergessen \\ Soltu / wonn es ist das dritte \\ Der luft das vierde / Das fúnfte domitte \\ Sattung lärung vnd das Seste \\ Zi̊nall gemütes / die habe veste \\ Wonn us den stúken můstu nèn \\ Der gesuntheit Regimen (Menge 1976:281 f.)
}

\section{Literaturverzeichnis}

\section{Primärliteratur:}

FERCKel, Christoph (Hrsg.) (1918): Ein Gesundheitsregiment für Herzog Albrecht von Österreich aus dem 14. Jahrhundert. In: Sudhoffs Archiv für Geschichte der Medizin, Nr. 11, S. 5-20.

Feyl, Anita (1963): Das Kochbuch Meister Eberhards. Ein Beitrag zur altdeutschen Fachliteratur. Diss. Freiburg im Breisgau.

Hagenmeyer, Christa (1972): Die Ordnung der Gesundheit für Rudolf von Hohenberg. Untersuchungen zur diätetischen Fachprosa des Spätmittelalters mit kritischer Textausgabe. Diss. Heidelberg. 
Hagenmeyer, Christa (1995): Das Regimen Sanitatis Konrads von Eichstätt. Quellen - Texte Wirkungsgeschichte. Stuttgart.

Hagenmeyer, Christa (1995): Das ,Regimen vitae‘. In: Hagenmeyer, Christa (Hrsg.): Das Regimen Sanitatis Konrads von Eichstätt. Quellen - Texte - Wirkungsgeschichte. Stuttgart, S. 193-222.

Hagenmeyer, Christa (1995): Das ,Büchlein der Gesundheit‘. In: HagenMeyer, Christa (Hrsg.): Das Regimen Sanitatis Konrads von Eichstätt. Quellen - Texte - Wirkungsgeschichte. Stuttgart, S. 223-254.

KÜLz, Constantin / KüLz-Trosse, Emma (1908): Das Breslauer Arzneibuch. Sonderdruck Dresden. Liber pantegni (1539). In: Summi In Omni Philosophia Viri Constantini Africani. Basel, S. 98-142. Menge, Heinz H. (1976): Das Regimen Heinrich Laufenbergs: Textologische Untersuchung und Edition. Göppingen.

De RenzI, Salvatore (Hrsg.) (1852-59): Collectio Salernitana. 5 Bde. Neapel.

MöLLER, Reinhold (1963): Hiltgart von Hürnheim: Mittelhochdeutsche Prosaübersetzung des Seretum secretorum. Berlin.

,Regimen sanitatis ad inclitum dominum regem Aragonum“ (1996). In: GARCÍA-BALLESTER, Luis / Paniagua, Juan Antonio / McVaugh, Michael Rogers (Hrsg.): Arnaldi de Villanova Opera medica omnia. Bd. 10, Teil 1, S. 423-470.

STRAUSS, Peter (1963): Arnald von Villanova deutsch unter besonderer Berücksichtigung der Regel der Gesundheit. Diss. Heidelberg.

\section{Sekundärliteratur:}

Adamson, Melitta Weiss (1995): Medieval Dietetics. Food and Drink in Regimen Sanitatis Literature from 800 to 1400. Frankfurt a. M.; Berlin; Bern u. a.

HIRTH, Wolfgang (1966): Die Diätetik im Kochbuch des Küchenmeisters Eberhart von Landshut und eine deutsche Regel der Gesundheit nach Arnald de Villanova. In: Ostbairische Grenzmarken, Nr. 8, S. 273-281.

Schmitт, Wolfram (1973): Theorie der Gesundheit und Regimen sanitatis im Mittelalter. Habilitation Heidelberg.

SCHNELL, Bernhard (1998). Heinrich Laufenbergs ,Regimen“. In: Heinrich Laufenberg - Regimen der Gesundheit. Iatromathematisches Hausbuch. Michael Puff - Von den ausgebrannten Wässern. Farbmikrofiche-Edition der Handschrift Zürich, Zentralbibliothek, Ms. C 102b. Codices illuminati medii aevi 41. München, S. 7-27. 